\title{
Effects of Small-Scale Fluctuations of Neutrino Flux in Supernova Explosions
}

\author{
Hideki Madokoro, Tetsuya Shimizu, and Yuko Motizuki \\ RIKEN, Hirosawa 2-1, Wako 351-0198, Japan madokoro@postman.riken.go.jp
}

Summary. We examine effects of small-scale fluctuations with angle in the neutrino radiation in core-collapse supernova explosions. As the mode number of fluctuations increases, the results approach those of spherical explosion. We conclude that global anisotropy of the neutrino radiation is the most effective mechanism of increasing the explosion energy when the total neutrino luminosity is given.

\section{Introduction}

It has been turned out that most simulations of core-collapse supernova explosions with spherical symmetry fail to produce a successful explosion[1]. In addition, observations suggest that the ejecta of core-collapse supernova explosions are aspherical(e.g., [2]). These facts lead us to multidimensional simulations. In 2-D and $3-\mathrm{D}$ simulations $[3,4,5,6,7,8,9,10,11]$, it has been shown that multidimensional effects, such as convection inside the proto-neutron star and convective overturn around the neutrino-heated region, increase the explosion energy and can trigger a successful explosion $[4,6,12]$.

When a proto-neutron star rotates, the neutrino flux is expected to be enhanced along the rotational (polar) axis. Janka and Mönchmeyer[13, 14] first discussed the possibility of aspherical neutrino emission from a rapidly rotating inner core. They argued that a neutrino flux along the polar axis might become three times greater than that on the equatorial plane.

Shimizu et al.[15, 9] proposed that the anisotropic neutrino radiation should play a crucial role in the explosion mechanism itself. They carefully investigated the effects of anisotropic neutrino radiation on the explosion energy. They found that only a few percent enhancement in the neutrino emission along the pole is sufficient to increase the explosion energy by a large factor, and leads to a successful explosion. They also found that this effect saturates around a certain degree of anisotropy. It should be noted here that the assumed rotational velocity of the inner core is very different between Janka et al.[13, 14] and Shimizu et al.[15, 9].

In the work of Shimizu et al.[9], they considered only a form of global anisotropy; the maximum peak in the neutrino flux distribution was located at the pole and the 
minimum at the equatorial plane. On the other hand, Burrows et al.[5] have suggested that the neutrino flux can fluctuate with angle and time due to gravitational oscillation on the surface of the proto-neutron star. In this work, we introduce such small-scale fluctuations in the neutrino flux in our numerical code by modifying the angular distribution of the neutrino flux. We aim to study the effects of these smallscale fluctuations on the shock position, the explosion energy, and the asymmetric explosion. Details are found in ref.[16].

\section{Numerical Code}

We perform 2-D simulations by solving hydrodynamic equations in spherical coordinates. A generalized Roe's method is employed to solve the hydrodynamic equations with general equations of state (EOSs). The details of our numerical technique, together with the EOS and the initial condition used, are described in the previous article[9]. In our study, we have improved the numerical code of Shimizu et al.[9]; the cells in the $\theta$-direction were shifted by half of the cell size[17] in order to avoid a numerical error near the pole, although the error was not serious for the investigation of the explosion energy.

In the present work, the local neutrino flux is assumed to be[16]

$$
l_{\nu}(r, \theta)=\frac{7}{16} \sigma T_{\nu}^{4} c_{1}\left(1+c_{2} \cos ^{2}\left(n_{\theta} \theta\right)\right) \frac{1}{r^{2}},
$$

where $\sigma$ is the Boltzmann constant, and $T_{\nu}$ is the temperature on the neutrinosphere. In equation (1), $n_{\theta}$ represents the number of waves in the $\theta$-direction. The case of $n_{\theta}=1$ corresponds to the global anisotropy, namely, no fluctuation. $c_{2}$ is a parameter which is related to the degree of anisotropy in the neutrino radiation. We see in equation (1) that the neutrino fluxes in the $x$ (equatorial) and $z$ (polar) directions become $l_{x} \equiv l_{\nu}\left(r, \theta=90^{\circ}\right) \propto c_{1}$ and $l_{z} \equiv l_{\nu}\left(r, \theta=0^{\circ}\right) \propto c_{1}\left(1+c_{2}\right)$, respectively. The degree of anisotropy $l_{z} / l_{x}$ is then represented as

$$
\frac{l_{z}}{l_{x}}=1+c_{2}
$$

The value of $c_{1}$ is calculated from $c_{2}$ and $n_{\theta}$ so as to adjust the total neutrino flux to that in the spherical model at the same $T_{\nu}$.

It should be noted here that the amplitude of fluctuations in the neutrino flux distribution for an observer far from the neutrinosphere and that on the neutrinoemitting surface are different. When we observe the neutrino flux far from the neutrinosphere, the local neutrino flux is seen as equation (1). On the other hand, the neutrino flux on the neutrino-emitting surface has a profile similar to equation (1) but the amplitude is different. In the latter, $c_{2}$ is replaced by $a$ where $a$ is a parameter which represents the degree of anisotropy of the neutrino flux on the neutrinosphere. It is preferable that we compare the results for the same value of $a$, since $a$ is more directly related to explosion dynamics. The value of $c_{2}$, therefore, is calculated from a given $a$, depending on $n_{\theta}$. Although it is difficult to calculate the exact relationship between $c_{2}$ and $a$, we can estimate it by assuming that the strength of the neutrino flux on the neutrinosphere is approximated by a profile of step function. This makes it possible to relate the value of $c_{2}$ to the value of $a$ for each $n_{\theta}$. For detail, see Madokoro et al.[16]. 
We set $a=0.31$ in this work. This value of $a$ is chosen in such a way that the value of $l_{z} / l_{x}$ for the global model $\left(n_{\theta}=1\right)$ becomes 1.10 . The values of $c_{2}$ for each fluctuation model $\left(n_{\theta}=3,5\right)$ are accordingly calculated. These are summarized in Table 1 . The neutrino temperature on the neutrino-emitting surface $T_{\nu}$ is assumed to be $4.70 \mathrm{MeV}$. In our simulation, we have 500 nonuniform radial zones which cover from 50 to $10000 \mathrm{~km}$ in radius. For $\theta$, we have 62 uniform angular zones from $\theta=0^{\circ}$ to $\theta=90^{\circ}$ with equatorial symmetry for $n_{\theta}=1$ and 3 . Note that we use a double value of the angular zones for $n_{\theta}=5$, which is different from that used in ref.[16].

\section{Results}

Figure 1 depicts the contour maps of the dimensionless entropy[9] distribution with the velocity fields for the three models at $t \sim 250 \mathrm{~ms}$ after the shock stall. The shock front is represented by the crowded contour lines at $r \sim 2000-3000 \mathrm{~km}$ for the model A1-T470, $r \sim 1600-2200 \mathrm{~km}$ for the model A3-T470, and $r \sim 1400 \mathrm{~km}$ for the model A5-T470. We see in Figure 1 that the shock front is largely distorted in a prolate form for the model A1-T470. This is because the neutrino heating along the pole is more intensive than that on the equatorial plane. Due to increased pressure in the locally heated matter near the polar axis, the shock front along the pole is pushed up, resulting in a prolate deformation. We find that the shock position becomes less extended than that of the global anisotropy, and the shock front approaches the spherical shape when the mode number of fluctuation increases. This trend is especially remarkable for the model A5-T470 in which the shock front is almost spherical.

Figure 2 shows the evolution of the explosion energy, as well as the thermal, kinetic, and gravitational energies for the three models. The energy gain for the case of $n_{\theta}=1$ is the highest among others at all stages of the explosion. It is also seen in Figure 2 that the explosion energy decreases as the mode number of fluctuations in the neutrino flux increases and finally approaches that of the spherical explosion.

Thus, we found that there are remarkable differences in the explosion energy depending on the mode number of the fluctuations. We also found that larger number of modes in the fluctuations makes the result closer to that of the spherical explosion. This is because any small-scale fluctuations on the neutrinosphere are greatly averaged out when the neutrino emission is observed far enough from the neutrino-emitting surface. Moreover, we found that a certain broad space is needed to be heated by neutrinos to revive the stalled shock wave rigorously and hence the global anisotropy $\left(n_{\theta}=1\right)$ is the most effective to increase the explosion energy. These results support the claim by Shimizu et al.[9].

\section{Conclusion}

We have investigated the effects of small-scale fluctuations in the neutrino flux on the core-collapse supernova explosion. The profile of the neutrino radiation field was specified taking its geometric effects into account. Since the small-scale fluctuations are averaged out for radiative and hydrodynamic reasons, the results of the fluctuation models become closer to that of the spherical explosion. Consequently, the 
global anisotropy is the most effective mechanism in increasing the explosion energy when $L_{\nu}$ is given. This supports the claim made by Shimizu et al.[9].

\begin{tabular}{|c|c|c|}
\hline Model & $n_{\theta}$ & $c_{2}$ \\
\hline \hline $\mathrm{A} 1-\mathrm{T} 470$ & 1 & 0.100 \\
\hline $\mathrm{A} 3-\mathrm{T} 470$ & 3 & 0.051 \\
\hline $\mathrm{A} 5-\mathrm{T} 470$ & 5 & 0.035 \\
\hline
\end{tabular}

Table 1. Simulated models.
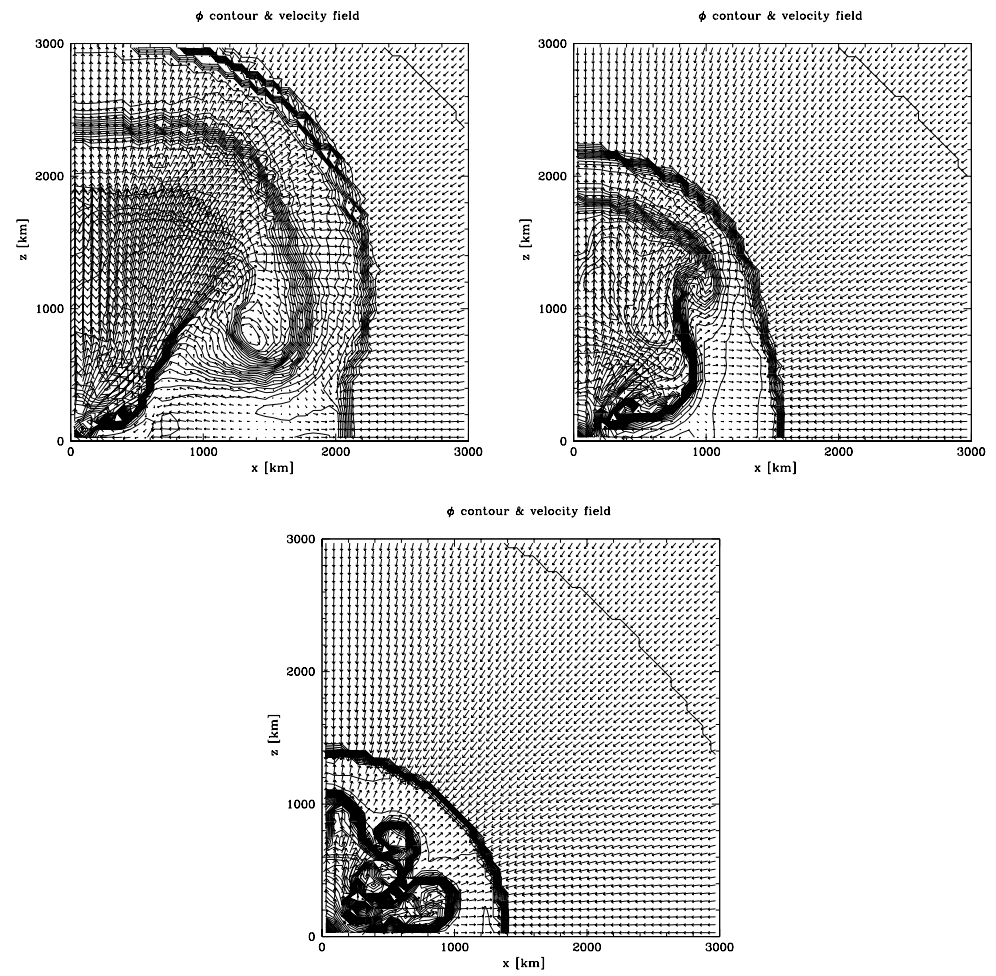

Fig. 1. Contour maps of the dimensionless entropy distribution and the velocity fields for the three models; top left: $n_{\theta}=1$ (model A1-T470) at $t=244 \mathrm{~ms}$, top right: $n_{\theta}=3$ (model A3-T470) at $t=254 \mathrm{~ms}$, bottom: $n_{\theta}=5($ model A5-T470) at $t=252 \mathrm{~ms}$. 


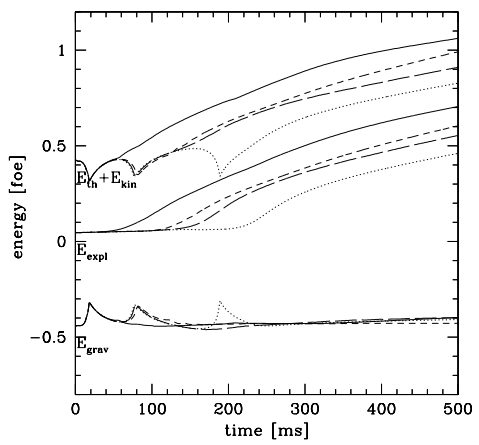

Fig. 2. Evolution of thermal and kinetic energy $\left(E_{\mathrm{th}}+E_{\mathrm{kin}}\right)$, gravitational energy $\left(E_{\text {grav }}\right)$ and explosion energy $\left(E_{\text {expl }}\right)$ for the three models; solid line: $n_{\theta}=1$, shortdashed line: $n_{\theta}=3$, long-dashed line: $n_{\theta}=5$. For comparison, the result of the spherical explosion at the same $T_{\nu}$ is also plotted (dotted line).

\section{References}

1. M. Liebendoerfer, A. Mezzacappa, F. -K. Thielemann, O. E. B. Messer, W. R. Hix, \& S. W. Bruenn: Phys. Rev. D 63, 103004 (2001)

2. L. Wang et al: ApJ 579, 671 (2002)

3. D. S. Miller, J. R. Wilson, \& R. W. Mayle: ApJ 415, 278 (1993)

4. M. Herant, W. Benz, W. R. Hix, C. L. Fryer, \& S. A. Colgate: ApJ 435, 339 (1994)

5. A. Burrows, J. Hayes, \& B. A. Fryxell: ApJ 450, 830 (1995)

6. H. -T. Janka, \& E. Müller: A \& A 306, 167 (1996)

7. A. Mezzacappa, A. C. Calder, S. W. Bruenn, J. M. Blondin, M. W. Guidry, M. R. Strayer, \& A. S. Umar: ApJ 495, 911 (1998)

8. C. L. Fryer, \& A. Heger: ApJ 541, 1033 (2000)

9. T. M. Shimizu, T. Ebisuzaki, K. Sato, \& S. Yamada: ApJ 552, 756 (2001)

10. C. L. Fryer, \& M. S. Warren: ApJL 574, L65 (2002)

11. K. Kifonidis, T. Plewa, H. -T. Janka, \& E. Müller: A \& A , submitted (astroph/0302239) (2003)

12. W. Keil, H. -T. Janka, \& E. Müller: ApJ 473, L111 (1996)

13. H. -T. Janka, \& R. Mönchmeyer: A \& A 209, L5 (1989)

14. H. -T. Janka, \& R. Mönchmeyer: A \& A 226, 69 (1989)

15. T. Shimizu, S. Yamada, \& K. Sato: ApJ 432, L119 (1994)

16. H. Madokoro, T. Shimizu, \& Y. Motizuki: ApJ 592, 1035 (2003)

17. T. M. Shimizu: Ph.D.thesis, Univ. Tokyo (1995) 